\title{
叔丁基过氧化氢介导的磷酸硒酯合成
}

\author{
张云倩 周晨凡 刘功清*
}

(南通大学药学院 南通 226001)

\begin{abstract}
摘要 磷酸硒酯化合物广泛应用于有机合成和药物发现等相关领域. 因此, 开发高效简便合成该类化合物的新方法受 到化学家的广泛关注. 报道了一种以叔丁基过氧化氢(TBHP)为氧化剂, 在温和的反应条件下实现了亚磷酸酯和二硒梄 偶联反应制备硒代磷酸酯方法. 与现有方法相比，该方法具有条件温和、底物适用范围广泛、产率高、且不需过渡金 属和强碱等优点.

关键词＼cjkstart叔丁基过氧化氢；二硒醚；亚磷酸酯；磷酸硒酯
\end{abstract}

\section{tert-Butyl Hydroperoxide-Mediated Synthesis of Phosphoroselenoates}

\author{
Zhang, Yunqian Zhou, Chenfan Liu, Gongqing* \\ (School of Pharmacy, Nantong University, Nantong 226001)
}

\begin{abstract}
Phosphoroselenoates have found widespread applications in the fields of organic synthesis and drug discovery. To this end, continuous efforts have been devoted to the development of novel and efficient methods for the synthesis of these compounds. A new method for the synthesis of phosphoroselenoates through oxidative coupling reaction of diselenides with phosphites by the utilization of $t$-butyl hydroperoxide (TBHP) as oxidant was herein developed. The distinct advantages of this protocol over all previous methods include mild reaction conditions, broad substrate scope, excellent yields, as well as transition metal- and base-free.
\end{abstract}

Keywords tert-butyl hydroperoxide; diselenide; phosphite; phosphoroselenoate

磷酸硒酯(phosphoroselenoate)骨架结构是一种重要 的有机结构基础单元，广泛存在于生物活性分子中 ${ }^{[1]}$ (图 1), 在有机合成中也具有较大的应用价值 ${ }^{[2]}$. 因此该 类化合物的合成研究得到化学家们的广泛关注. 早期合 成这类化合物的主要方法包括使用苯基卤化硒与亚磷 酸三烷基酯反应或者苯硒化锂与 $O, O$-二烷基膦酰氯反 应 ${ }^{[2 a, 3]}$. 但这些原料的制备需要使用毒性试剂如氯气、溴 素, 此外, 这些原料对空气敏感, 因此反应条件苛刻, 反应操作繁琐.

二硒醚化合物作为非常稳定且容易操作的硒源一 直是合成化学家从事有机硒化学研究的首选目标 ${ }^{[4]}$. 近 年来, 通过活化二硒醚化合物与亚磷酸酯反应来制备硒 代磷酸酯化合物报道较多. 研究表明过渡金属 $[16,5]$ 、强

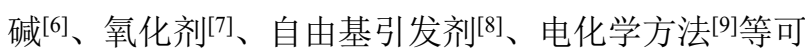

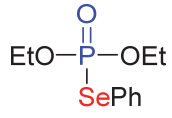

antimicrobial agent

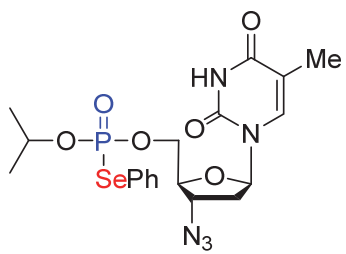

zidovudine (AZT) derivative (treatment of AIDS)<smiles>COc1ccc([Se]P(=O)(OC)O[Na])cc1</smiles>

antioxidant activity
图 1 含磷酸硒酯的生物活性分子

Figure 1 Bioactive molecules containing phosphoroselenoates 以活化断裂二硒醚化合物中 $\mathrm{Se}-\mathrm{Se}$ 键，原位生成活性

\footnotetext{
* Corresponding author. E-mail: gqliu@ntu.edu.cn

Received July 25, 2021; revised August 24, 2021; published online September 6, 2021.

Project supported by the Natural Science Foundation of Jiangsu Province (No. BK20170439), and the Science and Technology Plan Projects of Nantong City (Nos. JC2019102, JC2020072).

江苏省自然科学基金(No. BK20170439)和南通市科技(Nos. JC2019102, JC2020072)资助项目.
} 
的硒物种与亚磷酸二烷基酯反应合成硒代磷酸酯 (Scheme 1a). 尽管上述方法大大扩充了硒代磷酸酯化合 物的合成途径, 但这些方法大都使用等物质的量的二硒 醚，原子经济性不足; 强碱等苛刻反应条件限制了底物 适用范围; 此外, 由于碲代磷酸酯(phosphorotelluroate) 相对不稳定 ${ }^{[10]}$, 其合成方法研究相对较少. 因此, 继续 发展简洁、高效、通用的合成硒(碲)磷酸酯的方法仍有 必要. 本课题组在前期合成含硒化合物基础之上 ${ }^{[11]}$, 本 文利用廉价易得的叔丁基过氧化氢(TBHP)作为氧化剂 来源, 合成了硒代磷酸酯和碲代磷酸酯化合物(Scheme 1b).

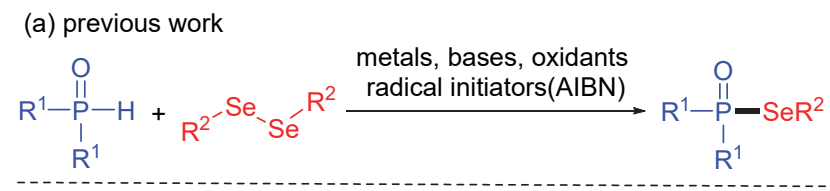

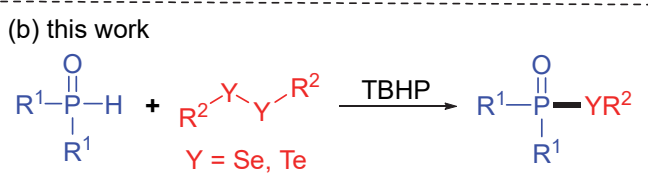

图式 1 硒代磷酸酯的合成 Scheme 1 Synthesis of phosphoroselenoates

\section{1 结果与讨论}

选用亚磷酸二甲酯(1a)与二苯基二硒醚 $(\mathbf{2 a})$ 作为模 板反应, 研究了氧化剂、溶剂和反应时间等因素对反应 结果的影响, 实验结果总结于表 1 . 从表 1 可以看出, 当 使用乙酸乙酯作为反应溶剂时, 不同种类的氧化剂对反 应收率有较为明显的影响. 高价碘化合物作为氧化剂 时, 室温条件下摚拌 $5 \mathrm{~h}$ 得到中等以下收率的目标产物 $O, O$-二甲基- $S e$-苯基磷酸酯(3a) (表 1, Entries 1 2). 无 机氧化剂过硫酸钾 $\left(\mathrm{K}_{2} \mathrm{~S}_{2} \mathrm{O}_{8}\right)$ 、过硫酸铵 $\left[\left(\mathrm{NH}_{4}\right)_{2} \mathrm{~S}_{2} \mathrm{O}_{8}\right]$ 和单 过硫酸氢钾(oxone)、五氧化二碘 $\left(\mathrm{I}_{2} \mathrm{O}_{5}\right)$ 分别以不同的产 率得到目标化合物 $3 \mathbf{a}$ (表 1 , Entries $3 \sim 6$ ). 为了提高反应 产率, 考察了有机氧化剂如间氯过氧苯甲酸 $(m \mathrm{CPBA})$ 、 TBHP 和氧气对反应的影响(表 1, Entries 7 9), 其中 TBHP 的效果最好(Entry 8). 随后以 TBHP 作为最优氧 化剂, 研究了不同极性的溶剂对反应的影响(表 1, Entries $10 \sim 15)$. 实验结果表明, 在使用二甲基亚砜 (DMSO)时反应效果最佳, 以 $88 \%$ 收率得到硒代磷酸酯 $3 \mathbf{a}$ (表 1, Entry 10). 这可能是由于 DMSO 不仅可以作为 溶剂使用, 而且可以作为氧化剂 ${ }^{[12]}$. 接着, 考察 TBHP 用量对反应的影响, 结果表明 2.5 equiv. 的 TBHP 是最佳 选择(表 1 , Entries 16 17). 之后将反应时间缩短为 $2 \mathrm{~h}$, 反应产率没有减少(表 1 , Entries 18～19). 对照实验证实 在没有 TBHP 存在下, 只检测到痕量的目标产物(表 1, Entry 20), 这些结果表明, TBHP 对反应发生是不可或缺
的. 因此最终确定优化反应条件为: 2.5 equiv.的 TBHP 作为氧化剂, DMSO 作为反应溶剂, 反应时间为 $2 \mathrm{~h}$.

表 1 反应条件的优化 ${ }^{a}$

Table 1 Optimization of reaction conditions

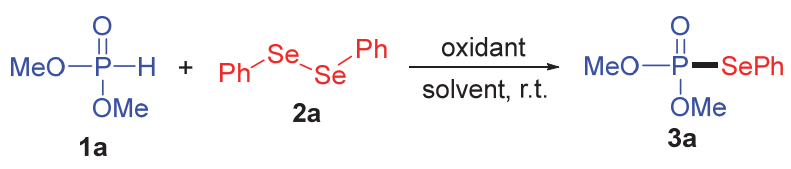

\begin{tabular}{cllcc}
\hline Entry & Oxidant & Solvent & Time/h & Isolated yield/\% \\
\hline 1 & PhIO & EtOAc & 5 & 43 \\
2 & ${\mathrm{PhI}(\mathrm{OAc})_{2}}_{2}$ & EtOAc & 5 & 35 \\
3 & $\mathrm{~K}_{2} \mathrm{~S}_{2} \mathrm{O}_{8}$ & EtOAc & 5 & 45 \\
4 & $\left(\mathrm{NH}_{4}\right)_{2} \mathrm{~S}_{2} \mathrm{O}_{8}$ & EtOAc & 5 & 40 \\
5 & $\mathrm{I}_{2} \mathrm{O}_{5}$ & EtOAc & 5 & 26 \\
6 & Oxone & EtOAc & 5 & 51 \\
7 & $m$ CPBA & EtOAc & 5 & 45 \\
8 & TBHP & EtOAc & 5 & 65 \\
9 & O2 & EtOAc & 5 & 30 \\
10 & TBHP & DMSO & 5 & 88 \\
11 & TBHP & DMF & 5 & 65 \\
12 & TBHP & EtOH & 5 & 55 \\
13 & TBHP & THF & 5 & 50 \\
14 & TBHP & Hexane & 5 & 56 \\
15 & TBHP & Acetone & 5 & 70 \\
$16^{b}$ & TBHP & DMSO & 5 & 76 \\
$17^{c}$ & TBHP & DMSO & 5 & 65 \\
18 & TBHP & DMSO & 2 & 89 \\
19 & TBHP & DMSO & 1 & 73 \\
20 & & DMSO & 2 & trace \\
\hline
\end{tabular}

${ }^{a}$ The reaction was performed with 1 a $(0.2 \mathrm{mmol}, 1$ equiv. $)$, diphenyl diselenide $2 \mathrm{a}(0.1 \mathrm{mmol}, 0.5$ equiv. $)$, oxidant $(0.5 \mathrm{mmol}, 2.5$ equiv. $)$ and solvent (1 $\mathrm{mL}) .{ }^{b} 0.6 \mathrm{mmol} \mathrm{TBHP} .{ }^{c} 0.4 \mathrm{mmol}$ TBHP.

在得到最优反应条件后，对反应的普适性进行了考 察. 首先, 对亚磷酸酯的适用性进行了考察(表 2). 结果 表明各种烷基亚磷酸酯都可以与二苯基二硒醚发生反 应，以较高的收率得到 $\mathrm{O}, \mathrm{O}$-二烷基- $\mathrm{Se}$-苯基磷酸酯 (3a 3g). 烷基亚磷酸酯中烷基链的大小对收率影响不 大. 例如, 位阻较小的亚磷酸二甲酯和具有较大位阻的 亚磷酸二叔丁酯参与反应时, 相应产物的产率分别为 $89 \%$ 和 $78 \%$. 另外, 亚磷酸二苯酯也能参与该反应的转 化, 得到的产率为 $88 \%(3 \mathrm{~h})$. 值得注意的是, 二苯氧磷6-氧化物和二苯基氧磷也具有良好的耐受性, 分别得到 产率为 $63 \%$ 和 $75 \%$ 的产物 $(\mathbf{3 i} \sim \mathbf{3} \mathbf{j})$.

随后，在最优反应条件下进一步考察了二硒醚底物 的普适性, 实验结果总结于表 3. 总体而言, 二芳基二 硒醚的底物(70\% 93\%, 4a 4h) 较二烷基二硒醚底物 $(61 \% \sim 77 \%, 4 \mathbf{i} \sim 4 p)$ 更利于反应的进行, 产率偏高. 二 芳基二硒醚底物中芳环上电子效应对反应影响不大，例 如当二苯基二硒醚底物的苯环上连有给电子的烷基、烷 氧基或吸电子基团卤素时，均可以较好的产率得到目标 
产物 $(4 \mathrm{a} \sim 4 \mathrm{~h})$. 二烷基二硒醚底物应用于当前反应体系 时, 以良好的产率得到各种 $\mathrm{O}, \mathrm{O}$-二苯基-Se-烷基磷酸酯 $(4 \mathbf{i} \sim 4 p)$. 最后, 该体系对二碲醚底物同样适用, 以中等 产率得到相应的碲代磷酸酯 $(\mathbf{4 q} \sim \mathbf{4 t})$.

表 2 亚磷酸酯底物的拓展 $a$

Table 2 Expansion of phosphites

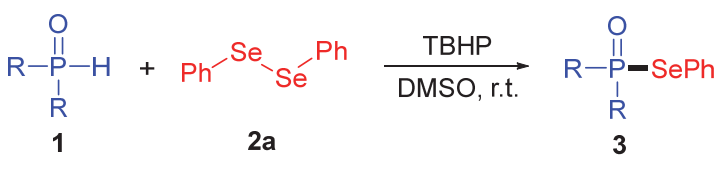<smiles>COP(=O)(OC)[Se]c1ccccc1</smiles>

3a $89 \%$

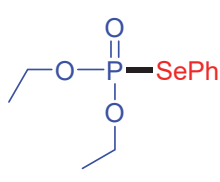

3b $91 \%$

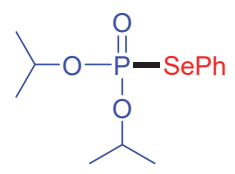

3c $81 \%$<smiles>CC(C)(C)OP(=O)(OC(C)(C)C)[Se]c1ccccc1</smiles>

3d $78 \%$<smiles>O=P(OCc1ccccc1)(OCc1ccccc1)Sc1ccccc1</smiles>

3g $81 \%$<smiles>O=P(c1ccccc1)(c1ccccc1)c1ccccc1</smiles>

3j $75 \%$
${ }^{a}$ The reaction was performed with $\mathbf{1}(0.2 \mathrm{mmol}, 1$ equiv.), diphenyl diselenide 2a ( $0.1 \mathrm{mmol}, 0.5$ equiv.), TBHP ( $0.5 \mathrm{mmol}, 2.5$ equiv.) and DMSO $(1 \mathrm{~mL}), 2$ h, r.t.

为了更好地了解反应历程, 开展了如 Scheme 2 所 示的对照实验. 首先, 反应体系中添加了 3 equiv. 自由基 捕获剂 2,2,6,6-四甲基-1-哌啶氧基(TEMPO). 然而并未 观察到目标化合物 3a 的产率有明显的下降(Scheme 2a), 这一现象表明该反应过程中可能没有自由基参与. 众所 周知, 二苯基二硒醚容易被氧化剂氧化生成苯硒基阳离 子 $\left(\mathrm{PhSe}^{+}\right)^{[13]}$, 我们推测过量的 TBHP 也可以将二硒醚 氧化产生 $\mathrm{PhSe}^{+}$. 为了验证这一假设, 在标准条件下向 反应体系中加入了 3 equiv. 的富电子的竞争底物吲哚和 1,3,5-三甲氧基苯 ${ }^{[14]}$, 用来捕获反应过程中任何可能形 成的 $\mathrm{PhSe}^{+}$. 正如预期那样，我们分别以 $35 \%$ 和 $43 \%$ 的 分离产率得到了相应的吲哚硒醚 5 和苯基硒醚 6 (Scheme 2b, 2c).

基于以上实验结果和文献报道, 推测了可能的反应 机理. 如 Scheme 3 所示, 首先, TBHP 与 $\mathrm{R}_{2} \mathrm{Se}_{2}$ 反应生成
亚硒酸 $\mathbf{A}(\mathrm{RSeOH})$ 和酸酐 $\mathbf{B}^{[15]}$. 极不稳定的中间体 $\mathbf{A}$ 通 过自缩合过程很容易转化为酸酐 $\mathbf{B}^{[16]}$, 亚磷酸酯亲核进 攻酸酐 $\mathbf{B}$ 生成磷酸硒酯和亚硒酸 $\mathbf{A}$, 后者脱水得到酸酐 $\mathbf{B}$ 与亚磷酸酯反应得到目标化合物.

表 3 二硫族属化合物的拓展 ${ }^{a}$

Table 3 Expansion of dichalcogenides
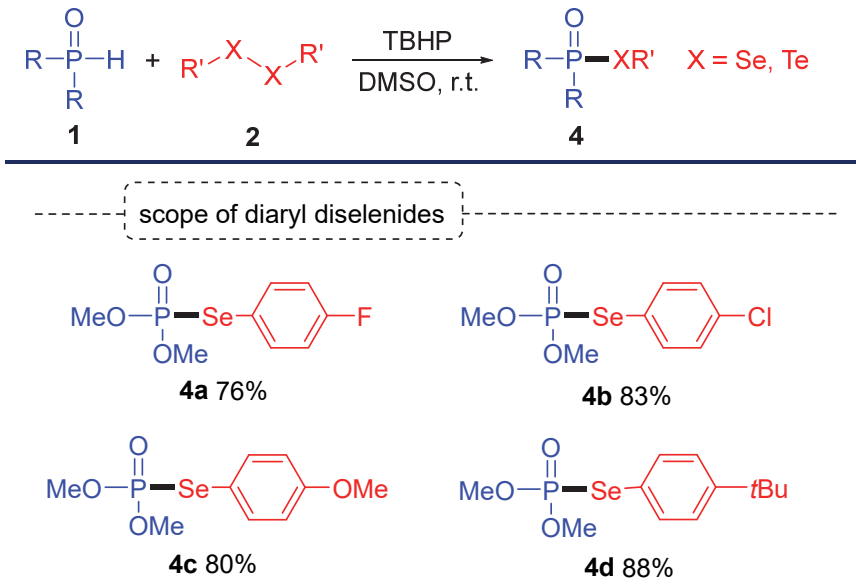<smiles>COP(=O)(OC)Oc1ccc(OC(F)(F)F)cc1</smiles>

4e $91 \%$

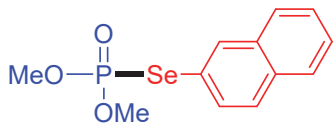

4f $93 \%$<smiles>COP(=O)(OC)Oc1cccs1</smiles>
$4 \mathrm{~g} 70 \%$

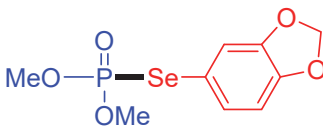

4h $77 \%$

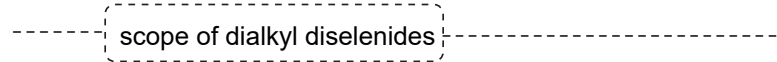

4i $71 \%$ 4j $60 \%$ 4k $64 \%$<smiles>CCCCCCC[Se]P(=O)(Oc1ccccc1)OP(=O)(O)OP(=O)([Se][Ge])[Se]C1CCCCC1</smiles>

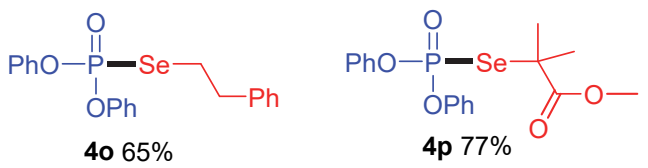

scope of ditellurides
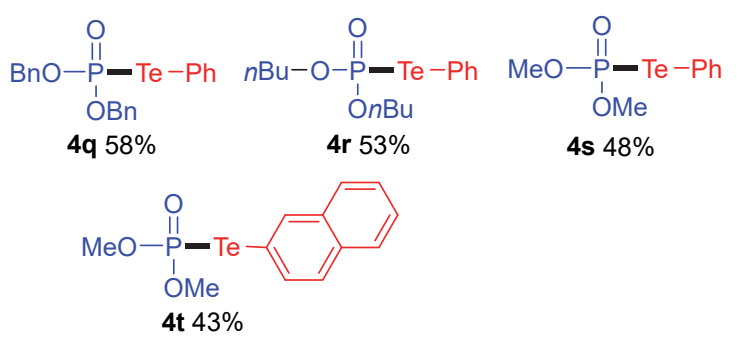

${ }^{a}$ The reaction was performed with 1 ( $0.2 \mathrm{mmol}, 1$ equiv.), diselenide 2 (0.1 mmol, 0.5 equiv. $)$ TBHP ( $0.5 \mathrm{mmol}, 2.5$ equiv. $)$ and DMSO $(1 \mathrm{~mL}), 2$ h, r.t. 

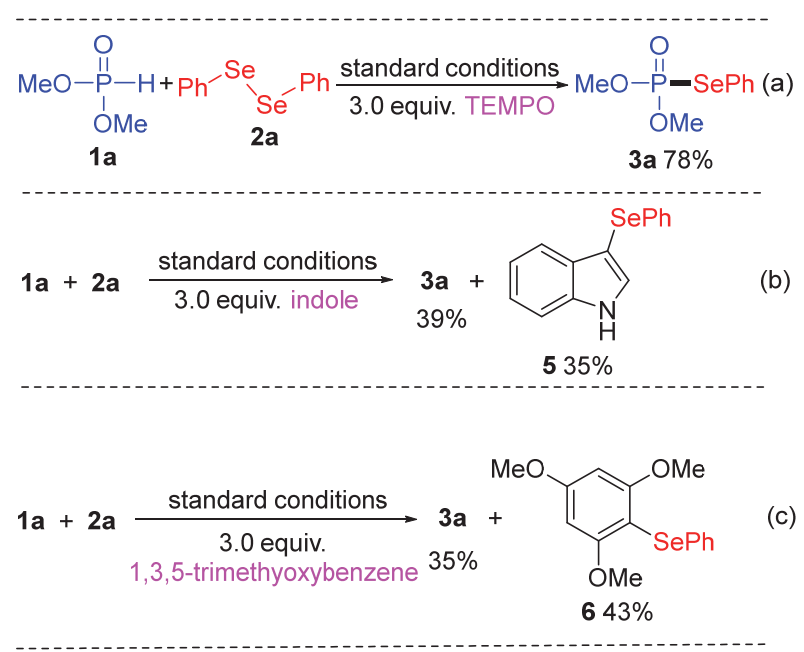

图式 2 对照实验

Scheme 2 Control experiments

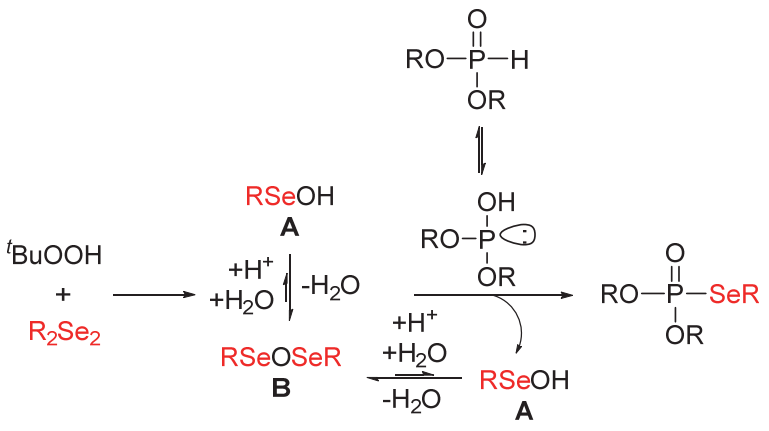

图式 3 可能的反应机理

Scheme 3 Proposed mechanism

\section{2 结论}

本文利用廉价易得的 TBHP 作为氧化剂, 商业化的 亚磷酸酯和二硒/碲醚为原料, 实现了一系列硒代磷酸 酯和碲代磷酸酯化合物的合成. 该方法可在空气条件下 操作, 对水和氧气不敏感, 反应条件温和, 操作简单, 收率高以及官能团兼容性好. 与以往的合成方法相比, 二硒/碲醚的用量大大减少，且不需要昂贵的过渡金属、 不需要添加强碱. 为硒基磷酸酯和碲基磷酸酯类化合物 开拓了新的合成路线和方法, 具有良好的应用潜力和研 究价值.

\section{3 实验部分}

\section{1 仪器与试剂}

所有实验药品均为市售分析纯级试剂, 未经进一步 处理直接使用. 柱层析使用 $200 \sim 300$ 目硅胶. 核磁共振 波谱均在德国布鲁克 400 兆核磁共振仪测得, 内标为三 甲基硅烷, 氛代氯仿为溶剂. 高分辨质谱测定采用安捷 伦公司 6210 型液质联用仪. WRS-1S 数字熔点仪, 温度 未校正. 亚磷酸酯、二茶基二硒醚和二苯基二碲醚购买
于国产试剂公司，二芳基二硒醚和二烷基二硒醚按照文 献方法合成 ${ }^{[17]}$.

\section{2 实验方法}

在反应瓶中依次加入二硒(碲)醚 $(0.10 \mathrm{mmol}, 0.50$ equiv.), 亚磷酸酯( $0.20 \mathrm{mmol}, 1.00$ equiv.), TBHP (0.50 mmol, 2.50 equiv.), DMSO (1 mL), 室温下搅拌 $2 \mathrm{~h}$, 薄 层色谱(TLC)检测反应完全后, 反应液加入 $10 \mathrm{~mL}$ 水, 用二氯甲烷萃取 3 次, 合并有机相后用无水硫酸钠干 燥、过滤，减压浓缩除去反应溶剂，残余物经硅胶柱层 析纯化, 使用石油醚和乙酸乙酯的混合物 $(V: V=15$ : $1 \sim 1: 1$ )作为洗脱剂, 得到目标化合物.

$O, O$-二甲基- $S e$-苯基磷酸酯 $(\mathbf{3 a})^{[7 b]}$ : 洗脱剂: $V$ (石油 醚) $: V($ 乙酸乙酯 $)=4: 1$, 无色油状液体. $47 \mathrm{mg}$, 产率 89\%. ${ }^{1} \mathrm{H}$ NMR $\left(400 \mathrm{MHz}, \mathrm{CDCl}_{3}\right) \delta: 7.56(\mathrm{~d}, J=6.7 \mathrm{~Hz}$, $2 \mathrm{H}), 7.44 \sim 6.97(\mathrm{~m}, 3 \mathrm{H}), 3.73(\mathrm{~s}, 3 \mathrm{H}), 3.69(\mathrm{~s}, 3 \mathrm{H}) ;{ }^{13} \mathrm{C}$ NMR $\left(100 \mathrm{MHz}, \mathrm{CDCl}_{3}\right) \delta: 134.6\left(\mathrm{~d}, J_{\mathrm{C}-\mathrm{P}}=4.7 \mathrm{~Hz}\right), 128.6$ $\left(\mathrm{d}, J_{\mathrm{C}-\mathrm{P}}=2.2 \mathrm{~Hz}\right), 127.9\left(\mathrm{~d}, J_{\mathrm{C}-\mathrm{P}}=2.7 \mathrm{~Hz}\right), 122.2\left(\mathrm{~d}, J_{\mathrm{C}-\mathrm{P}}=\right.$ $8.5 \mathrm{~Hz}), 53.0\left(\mathrm{~d}, J_{\mathrm{C}-\mathrm{P}}=5.7 \mathrm{~Hz}\right) ;{ }^{31} \mathrm{P}$ NMR $(162 \mathrm{MHz}$, $\left.\mathrm{CDCl}_{3}\right) \delta: 21.8$.

$O, O$-二乙基- $S e$-苯基磷酸酯 $(\mathbf{3 b})^{[9 \mathrm{a}]}$ : 洗脱剂: $V$ (石油 醚) $: V$ (乙酸乙酯 $)=5: 1$, 无色油状液体. $53 \mathrm{mg}$, 产率 91\%. ${ }^{1} \mathrm{H}$ NMR (400 MHz, $\left.\mathrm{CDCl}_{3}\right) \delta: 7.66 \sim 7.63(\mathrm{~m}, 2 \mathrm{H})$, $7.47 \sim 7.17(\mathrm{~m}, 3 \mathrm{H}), 4.40 \sim 3.75(\mathrm{~m}, 4 \mathrm{H}), 1.31(\mathrm{t}, J=7.1$ $\mathrm{Hz}, 6 \mathrm{H}) ;{ }^{13} \mathrm{C}$ NMR $\left(100 \mathrm{MHz}, \mathrm{CDCl}_{3}\right) \delta: 134.5\left(\mathrm{~d}, J_{\mathrm{C}-\mathrm{P}}=\right.$ $4.6 \mathrm{~Hz}), 128.5\left(\mathrm{~d}, J_{\mathrm{C}-\mathrm{P}}=2.1 \mathrm{~Hz}\right), 127.8\left(\mathrm{~d}, J_{\mathrm{C}-\mathrm{P}}=2.6 \mathrm{~Hz}\right)$, $122.7\left(\mathrm{~d}, J_{\mathrm{C}-\mathrm{P}}=8.5 \mathrm{~Hz}\right), 62.8\left(\mathrm{~d}, J_{\mathrm{C}-\mathrm{P}}=5.9 \mathrm{~Hz}\right), 14.9(\mathrm{~d}$, $\left.J_{\mathrm{C}-\mathrm{P}}=7.4 \mathrm{~Hz}\right) ;{ }^{31} \mathrm{P} \mathrm{NMR}\left(162 \mathrm{MHz}, \mathrm{CDCl}_{3}\right) \delta: 18.0$.

$O, O$-二异丙基- $S e$ - 苯基磷酸酯(3c) ${ }^{[7 b]}$ : 洗脱剂: $V$ (石 油醚) $: V$ (乙酸乙酯 $)=5: 1$, 无色油状液体. $52 \mathrm{mg}$, 产 率 $81 \%$. ${ }^{1} \mathrm{H}$ NMR $\left(400 \mathrm{MHz}, \mathrm{CDCl}_{3}\right) \delta: 7.59$ (d, $J=8.1$ $\mathrm{Hz}, 2 \mathrm{H}), 7.33 \sim 7.17(\mathrm{~m}, 3 \mathrm{H}), 4.89 \sim 4.57(\mathrm{~m}, 2 \mathrm{H}), 1.26(\mathrm{~d}$, $J=6.2 \mathrm{~Hz}, 6 \mathrm{H}), 1.18(\mathrm{~d}, J=6.2 \mathrm{~Hz}, 6 \mathrm{H}) ;{ }^{13} \mathrm{C}$ NMR $(100$ $\left.\mathrm{MHz}, \mathrm{CDCl}_{3}\right) \delta: 134.1\left(\mathrm{~d}, J_{\mathrm{C}-\mathrm{P}}=5.0 \mathrm{~Hz}\right), 128.3\left(\mathrm{~d}, J_{\mathrm{C}-\mathrm{P}}=\right.$ $1.9 \mathrm{~Hz}), 127.5\left(\mathrm{~d}, J_{\mathrm{C}-\mathrm{P}}=2.5 \mathrm{~Hz}\right), 123.5\left(\mathrm{~d}, J_{\mathrm{C}-\mathrm{P}}=8.3 \mathrm{~Hz}\right)$, $72.1\left(\mathrm{~d}, J_{\mathrm{C}-\mathrm{P}}=6.6 \mathrm{~Hz}\right), 22.9\left(\mathrm{~d}, J_{\mathrm{C}-\mathrm{P}}=3.8 \mathrm{~Hz}\right), 22.5(\mathrm{~d}$, $\left.J_{\mathrm{C}-\mathrm{P}}=5.9 \mathrm{~Hz}\right) ;{ }^{31} \mathrm{P} \mathrm{NMR}\left(162 \mathrm{MHz}, \mathrm{CDCl}_{3}\right) \delta: 14.7$.

$O, O$-二叔丁基- $S e$-苯基磷酸酯(3d)：洗脱剂：V(石油 醚)： $V($ 乙酸乙酯 $)=3: 1$, 黄色固体. $54 \mathrm{mg}$, 产率 $78 \%$, m.p. $49 \sim 50{ }^{\circ} \mathrm{C} ;{ }^{1} \mathrm{H}$ NMR $\left(400 \mathrm{MHz}, \mathrm{CDCl}_{3}\right) \delta: 7.62(\mathrm{~d}$, $J=8.0 \mathrm{~Hz}, 2 \mathrm{H}), 7.46 \sim 7.02(\mathrm{~m}, 3 \mathrm{H}), 1.39(\mathrm{~s}, 18 \mathrm{H}) ;{ }^{13} \mathrm{C}$ $\operatorname{NMR}\left(100 \mathrm{MHz}, \mathrm{CDCl}_{3}\right) \delta: 134.7\left(\mathrm{~d}, J_{\mathrm{C}-\mathrm{P}}=4.7 \mathrm{~Hz}\right), 128.0$ $\left(\mathrm{d}, J_{\mathrm{C}-\mathrm{P}}=2.2 \mathrm{~Hz}\right), 127.2\left(\mathrm{~d}, J_{\mathrm{C}-\mathrm{P}}=2.7 \mathrm{~Hz}\right), 124.9\left(\mathrm{~d}, J_{\mathrm{C}-\mathrm{P}}=\right.$ $8.9 \mathrm{~Hz}), 84.4\left(\mathrm{~d}, J_{\mathrm{C}-\mathrm{P}}=10.4 \mathrm{~Hz}\right), 29.2\left(\mathrm{~d}, J_{\mathrm{C}-\mathrm{P}}=4.4 \mathrm{~Hz}\right) ;{ }^{31} \mathrm{P}$ NMR (162 MHz, $\left.\mathrm{CDCl}_{3}\right) \delta: 5.0$. HRMS (ESI) calcd for $\mathrm{C}_{14} \mathrm{H}_{23} \mathrm{O}_{3} \mathrm{PSe}[\mathrm{M}+\mathrm{H}]^{+}$351.0623, found 351.0633 . 
$O, O$-二正丁基-Se-苯基磷酸酯 $(\mathbf{3 e})^{[7 b]}$ : 洗脱剂: $V($ 石 油醚) $: V$ (乙酸乙酯 $)=5: 1$, 无色油状液体. $51 \mathrm{mg}$, 产 率 73\%. ${ }^{1} \mathrm{H}$ NMR $\left(400 \mathrm{MHz}, \mathrm{CDCl}_{3}\right) \delta: 7.57(\mathrm{~d}, J=8.4$ $\mathrm{Hz}, 2 \mathrm{H}), 7.40 \sim 7.07(\mathrm{~m}, 3 \mathrm{H}), 4.31 \sim 3.78(\mathrm{~m}, 4 \mathrm{H}), 1.55$ $(\mathrm{dq}, J=8.2,6.6 \mathrm{~Hz}, 4 \mathrm{H}), 1.38 \sim 1.21(\mathrm{~m}, 4 \mathrm{H}), 0.82(\mathrm{t}, J=$ $7.4 \mathrm{~Hz}, 6 \mathrm{H}) ;{ }^{13} \mathrm{C}$ NMR $\left(100 \mathrm{MHz}, \mathrm{CDCl}_{3}\right) \delta: 134.5(\mathrm{~d}$, $\left.J_{\mathrm{C}-\mathrm{P}}=4.7 \mathrm{~Hz}\right), 128.4\left(\mathrm{~d}, J_{\mathrm{C}-\mathrm{P}}=2.1 \mathrm{~Hz}\right), 127.7\left(\mathrm{~d}, J_{\mathrm{C}-\mathrm{P}}=2.5\right.$ $\mathrm{Hz}), 122.8\left(\mathrm{~d}, J_{\mathrm{C}-\mathrm{P}}=8.3 \mathrm{~Hz}\right), 66.5\left(\mathrm{~d}, J_{\mathrm{C}-\mathrm{P}}=6.5 \mathrm{~Hz}\right), 31.0$ $\left(\mathrm{d}, J_{\mathrm{C}-\mathrm{P}}=7.3 \mathrm{~Hz}\right), 17.7,12.5 ;{ }^{31} \mathrm{P} \mathrm{NMR}\left(162 \mathrm{MHz}, \mathrm{CDCl}_{3}\right)$ $\delta: 18.0$.

$O, O$-二异丁基- $S e$-苯基磷酸酯(3f $)^{[\mathrm{aa}]}$ : 洗脱剂: $V($ 石 油醚) $: V$ (乙酸乙酯 $)=5: 1$, 黄色油状液体. $50 \mathrm{mg}$, 产 率 72\%. ${ }^{1} \mathrm{H}$ NMR $\left(400 \mathrm{MHz}, \mathrm{CDCl}_{3}\right) \delta: 7.58(\mathrm{~d}, J=6.6$ $\mathrm{Hz}, 2 \mathrm{H}), 7.38 \sim 7.11(\mathrm{~m}, 3 \mathrm{H}), 3.96 \sim 3.64(\mathrm{~m}, 4 \mathrm{H}), 1.85$ (dp, $J=13.3,6.7 \mathrm{~Hz}, 2 \mathrm{H}), 0.83(\mathrm{~d}, J=1.6 \mathrm{~Hz}, 6 \mathrm{H}), 0.82$ $(\mathrm{d}, J=1.6 \mathrm{~Hz}, 6 \mathrm{H}) ;{ }^{13} \mathrm{C}$ NMR $\left(100 \mathrm{MHz}, \mathrm{CDCl}_{3}\right) \delta: 134.5$ $\left(\mathrm{d}, J_{\mathrm{C}-\mathrm{P}}=4.7 \mathrm{~Hz}\right), 128.4\left(\mathrm{~d}, J_{\mathrm{C}-\mathrm{P}}=1.9 \mathrm{~Hz}\right), 127.7\left(\mathrm{~d}, J_{\mathrm{C}-\mathrm{P}}=\right.$ $2.5 \mathrm{~Hz}), 122.7\left(\mathrm{~d}, J_{\mathrm{C}-\mathrm{P}}=8.5 \mathrm{~Hz}\right), 72.6\left(\mathrm{~d}, J_{\mathrm{C}-\mathrm{P}}=7.0 \mathrm{~Hz}\right)$, $27.9\left(\mathrm{~d}, J_{\mathrm{C}-\mathrm{P}}=7.6 \mathrm{~Hz}\right), 17.66,17.65 ;{ }^{31} \mathrm{P}$ NMR $(162 \mathrm{MHz}$, $\left.\mathrm{CDCl}_{3}\right) \delta: 17.8$.

$O, O$-二芐基- $S e$-苯基磷酸酯 $(\mathbf{3 g})^{[7 b]}$ : 洗脱剂: $V($ 石油 醚) $: V$ (乙酸乙酯 $)=3: 1$. 黄色油状液体. $169 \mathrm{mg}$, 产率 81\%. ${ }^{1} \mathrm{H}$ NMR (400 MHz, $\left.\mathrm{CDCl}_{3}\right) \delta: 7.48(\mathrm{~d}, J=8.1 \mathrm{~Hz}$, $2 \mathrm{H}), 7.30 \sim 7.21(\mathrm{~m}, 7 \mathrm{H}), 7.21 \sim 7.11(\mathrm{~m}, 6 \mathrm{H}), 5.07 \sim 4.98$ $(\mathrm{m}, 4 \mathrm{H}) ;{ }^{13} \mathrm{C}$ NMR $\left(100 \mathrm{MHz}, \mathrm{CDCl}_{3}\right) \delta: 134.8\left(\mathrm{~d}, J_{\mathrm{C}-\mathrm{P}}=\right.$ $4.7 \mathrm{~Hz}), 134.2\left(\mathrm{~d}, J_{\mathrm{C}-\mathrm{P}}=7.8 \mathrm{~Hz}\right), 128.5\left(\mathrm{~d}, J_{\mathrm{C}-\mathrm{P}}=2.2 \mathrm{~Hz}\right)$, $127.9\left(\mathrm{~d}, J_{\mathrm{C}-\mathrm{P}}=2.7 \mathrm{~Hz}\right), 127.52,127.50,127.0,122.2(\mathrm{~d}$, $\left.J_{\mathrm{C}-\mathrm{P}}=8.6 \mathrm{~Hz}\right), 68.1\left(\mathrm{~d}, J_{\mathrm{C}-\mathrm{P}}=6.1 \mathrm{~Hz}\right) ;{ }^{31} \mathrm{P}$ NMR $(162 \mathrm{MHz}$, $\left.\mathrm{CDCl}_{3}\right) \delta: 18.5$.

$O, O, S e$-三苯基磷酸酯(3h) ${ }^{[7 b]}$ : 洗脱剂: $V$ (石油醚) : $V($ 乙酸乙酯 $)=5: 1$, 黄色油状液体. $68 \mathrm{mg}$, 产率 $88 \%$. ${ }^{1} \mathrm{H}$ NMR $\left(400 \mathrm{MHz}, \mathrm{CDCl}_{3}\right) \delta: 7.55 \sim 7.37(\mathrm{~m}, 2 \mathrm{H})$, $7.33 \sim 7.14(\mathrm{~m}, 7 \mathrm{H}), 7.15 \sim 7.07(\mathrm{~m}, 6 \mathrm{H}) ;{ }^{13} \mathrm{C}$ NMR $(100$ $\left.\mathrm{MHz}, \mathrm{CDCl}_{3}\right) \delta: 149.2\left(\mathrm{~d}, J_{\mathrm{C}-\mathrm{P}}=8.6 \mathrm{~Hz}\right), 135.2\left(\mathrm{~d}, J_{\mathrm{C}-\mathrm{P}}=\right.$ $4.8 \mathrm{~Hz}), 128.7\left(\mathrm{~d}, J_{\mathrm{C}-\mathrm{P}}=1.3 \mathrm{~Hz}\right), 128.5\left(\mathrm{~d}, J_{\mathrm{C}-\mathrm{P}}=2.5 \mathrm{~Hz}\right)$, $128.3\left(\mathrm{~d}, J_{\mathrm{C}-\mathrm{P}}=2.9 \mathrm{~Hz}\right), 124.6\left(\mathrm{~d}, J_{\mathrm{C}-\mathrm{P}}=1.6 \mathrm{~Hz}\right), 121.6(\mathrm{~d}$, $\left.J_{\mathrm{C}-\mathrm{P}}=9.1 \mathrm{~Hz}\right), 119.6\left(\mathrm{~d}, J_{\mathrm{C}-\mathrm{P}}=5.1 \mathrm{~Hz}\right) ;{ }^{31} \mathrm{P}$ NMR $(162$ $\left.\mathrm{MHz}, \mathrm{CDCl}_{3}\right) \delta: 9.5$.

6-苯硒基-二苯并 $[c, e][1,2]$ 氧代磷酸甘油酯-6-氧化 物 $(3 \mathbf{i})^{[9 a]}$ : 洗脱剂: $V$ (石油醚) $: V($ 乙酸乙酯 $)=5: 1$, 黄 色固体. $47 \mathrm{mg}$, 产率 $63 \%$, m.p. $140 \sim 141{ }^{\circ} \mathrm{C}$; ${ }^{1} \mathrm{H}$ NMR $\left(400 \mathrm{MHz}, \mathrm{CDCl}_{3}\right) \delta: 7.80(\mathrm{dd}, J=14.9,7.7 \mathrm{~Hz}, 1 \mathrm{H}), 7.69$ (dd, $J=8.1,6.5 \mathrm{~Hz}, 1 \mathrm{H}), 7.64 \sim 7.50(\mathrm{~m}, 2 \mathrm{H}), 7.39$ (tdd, $J=7.5,3.6,1.0 \mathrm{~Hz}, 1 \mathrm{H}), 7.32 \sim 7.22(\mathrm{~m}, 1 \mathrm{H}), 7.13 \sim 7.02$ $(\mathrm{m}, 3 \mathrm{H}), 6.92(\mathrm{t}, J=7.6 \mathrm{~Hz}, 2 \mathrm{H}) ;{ }^{13} \mathrm{C}$ NMR $(100 \mathrm{MHz}$,
$\left.\mathrm{CDCl}_{3}\right) \delta: 149.3\left(\mathrm{~d}, J_{\mathrm{C}-\mathrm{P}}=9.8 \mathrm{~Hz}\right), 135.9\left(\mathrm{~d}, J_{\mathrm{C}-\mathrm{P}}=3.7 \mathrm{~Hz}\right)$, $134.9\left(\mathrm{~d}, J_{\mathrm{C}-\mathrm{P}}=7.5 \mathrm{~Hz}\right), 132.7\left(\mathrm{~d}, J_{\mathrm{C}-\mathrm{P}}=2.7 \mathrm{~Hz}\right), 129.5(\mathrm{t}$, $\left.J_{\mathrm{C}-\mathrm{P}}=5.5 \mathrm{~Hz}\right), 128.0\left(\mathrm{dd}, J_{\mathrm{C}-\mathrm{P}}=5.2,2.7 \mathrm{~Hz}\right), 127.4(\mathrm{~d}$, $\left.J_{\mathrm{C}-\mathrm{P}}=15.0 \mathrm{~Hz}\right), 125.6,124.4,123.9,123.9,123.7,122.2(\mathrm{~d}$, $J=11.2 \mathrm{~Hz}), 120.9\left(\mathrm{~d}, J_{\mathrm{C}-\mathrm{P}}=7.1 \mathrm{~Hz}\right), 120.8\left(\mathrm{~d}, J_{\mathrm{C}-\mathrm{P}}=11.7\right.$ $\mathrm{Hz}), 119.0\left(\mathrm{~d}, J_{\mathrm{C}-\mathrm{P}}=7.0 \mathrm{~Hz}\right) ;{ }^{31} \mathrm{P} \mathrm{NMR}\left(162 \mathrm{MHz}, \mathrm{CDCl}_{3}\right)$ $\delta: 31.4$.

$S e$-苯基二苯基氧膦 $(\mathbf{3} \mathbf{j})^{[9 \mathrm{a}]}$ : 洗脱剂: $V$ (石油醚)： $V($ 乙酸乙酯 $)=4: 1$, 无色油状液体. $54 \mathrm{mg}$, 产率 $75 \%$. ${ }^{1} \mathrm{H}$ NMR $\left(400 \mathrm{MHz}, \mathrm{CDCl}_{3}\right) \delta: 7.87 \sim 7.64(\mathrm{~m}, 4 \mathrm{H})$, $7.51 \sim 7.26(\mathrm{~m}, 8 \mathrm{H}), 7.16(\mathrm{t}, J=8.0 \mathrm{~Hz}, 1 \mathrm{H}), 7.07(\mathrm{t}, J=$ $7.5 \mathrm{~Hz}, 2 \mathrm{H}) ;{ }^{13} \mathrm{C}$ NMR $\left(100 \mathrm{MHz}, \mathrm{CDCl}_{3}\right) \delta: 136.3(\mathrm{~d}$, $\left.J_{\mathrm{C}-\mathrm{P}}=3.3 \mathrm{~Hz}\right), 133.9,133.0,132.3\left(\mathrm{~d}, J_{\mathrm{C}-\mathrm{P}}=3.3 \mathrm{~Hz}\right), 131.4$ $\left(\mathrm{d}, J_{\mathrm{C}-\mathrm{P}}=10.6 \mathrm{~Hz}\right), 129.3\left(\mathrm{~d}, J_{\mathrm{C}-\mathrm{P}}=1.7 \mathrm{~Hz}\right), 128.5(\mathrm{~d}$, $\left.J_{\mathrm{C}-\mathrm{P}}=13.2 \mathrm{~Hz}\right), 123.7\left(\mathrm{~d}, J_{\mathrm{C}-\mathrm{P}}=15.7 \mathrm{~Hz}\right) ;{ }^{31} \mathrm{P}$ NMR $(162$ $\left.\mathrm{MHz}, \mathrm{CDCl}_{3}\right) \delta: 40.0$.

$O, O$-二甲基- $S e$-对氟苯基磷酸酯(4a) ${ }^{[1 \mathrm{~d}]}$ : 洗脱剂: $V($ 石油醚 $): V($ 乙酸乙酯 $)=2: 1$, 黄色油状液体. $43 \mathrm{mg}$, 产率 76\%. ${ }^{1} \mathrm{H}$ NMR $\left(400 \mathrm{MHz}, \mathrm{CDCl}_{3}\right) \delta: 7.54(\mathrm{dd}, J=$ $8.8,5.2 \mathrm{~Hz}, 1 \mathrm{H}), 6.98 \sim 6.93(\mathrm{~m}, 2 \mathrm{H}), 3.74(\mathrm{~s}, 3 \mathrm{H}), 3.71(\mathrm{~s}$, $3 \mathrm{H}) ;{ }^{13} \mathrm{C}$ NMR $\left(100 \mathrm{MHz}, \mathrm{CDCl}_{3}\right) \delta: 162.3\left(\mathrm{dd}, J_{\mathrm{C}-\mathrm{F}}=\right.$ $\left.249.7, J_{\mathrm{C}-\mathrm{P}}=3.0 \mathrm{~Hz}\right), 136.7\left(\mathrm{dd}, J_{\mathrm{C}-\mathrm{P}}=8.3, J_{\mathrm{C}-\mathrm{P}}=4.4 \mathrm{~Hz}\right)$, $116.8\left(\mathrm{dd}, J_{\mathrm{C}-\mathrm{F}}=8.7, J_{\mathrm{C}-\mathrm{P}}=3.5 \mathrm{~Hz}\right), 115.9\left(\mathrm{dd}, J_{\mathrm{C}-\mathrm{F}}=21.9\right.$, $\left.J_{\mathrm{C}-\mathrm{P}}=2.3 \mathrm{~Hz}\right), 53.1\left(\mathrm{~d}, J_{\mathrm{C}-\mathrm{P}}=5.9 \mathrm{~Hz}\right) ;{ }^{19} \mathrm{~F}$ NMR $(376 \mathrm{MHz}$, $\left.\mathrm{CDCl}_{3}\right) \delta:-111.7\left(\mathrm{~d}, J_{\mathrm{P}-\mathrm{F}}=4.8 \mathrm{~Hz}\right) ;{ }^{31} \mathrm{P}$ NMR $(162 \mathrm{MHz}$, $\left.\mathrm{CDCl}_{3}\right) \delta: 21.5\left(\mathrm{~d}, J_{\mathrm{P}-\mathrm{F}}=4.8 \mathrm{~Hz}\right)$.

$O, O$-二甲基- $S e$-对氯苯基磷酸酯 $(\mathbf{4 b})^{[7 b]}$ : 洗脱剂: $V$ (石油醚) $: V$ (乙酸乙酯) $=3: 1$, 无色油状液体. $50 \mathrm{mg}$, 产率 83\%. ${ }^{1} \mathrm{H}$ NMR $\left(400 \mathrm{MHz}, \mathrm{CDCl}_{3}\right) \delta: 7.50(\mathrm{~d}, J=8.5$ $\mathrm{Hz}, 2 \mathrm{H}), 7.22(\mathrm{~d}, J=8.5 \mathrm{~Hz}, 3 \mathrm{H}), 3.74(\mathrm{~s}, 3 \mathrm{H}), 3.71$ (s, $3 \mathrm{H}) ;{ }^{13} \mathrm{C}$ NMR $\left(100 \mathrm{MHz}, \mathrm{CDCl}_{3}\right) \delta: 135.9\left(\mathrm{~d}, J_{\mathrm{C}-\mathrm{P}}=4.6\right.$ $\mathrm{Hz}), 134.5\left(\mathrm{~d}, J_{\mathrm{C}-\mathrm{P}}=3.1 \mathrm{~Hz}\right), 128.8\left(\mathrm{~d}, J_{\mathrm{C}-\mathrm{P}}=2.2 \mathrm{~Hz}\right)$, $120.3\left(\mathrm{~d}, J_{\mathrm{C}-\mathrm{P}}=8.8 \mathrm{~Hz}\right), 53.1\left(\mathrm{~d}, J_{\mathrm{C}-\mathrm{P}}=5.9 \mathrm{~Hz}\right) ;{ }^{31} \mathrm{P} \mathrm{NMR}$ $\left(162 \mathrm{MHz}, \mathrm{CDCl}_{3}\right) \delta: 21.2$.

$O, O$-二甲基-Se-对甲氧基苯基磷酸酯(4c) ${ }^{[1 \mathrm{~d}]}$ : 洗脱 剂: $V$ (石油醚) $: V$ (乙酸乙酯 $)=1: 1$, 黄色油状液体. 47 $\mathrm{mg}$, 产率 80\%. ${ }^{1} \mathrm{H}$ NMR (400 MHz, $\left.\mathrm{CDCl}_{3}\right) \delta: 7.46(\mathrm{~d}, J$ $=8.8 \mathrm{~Hz}, 2 \mathrm{H}), 6.78(\mathrm{~d}, J=8.8 \mathrm{~Hz}, 2 \mathrm{H}), 3.72(\mathrm{~s}, 3 \mathrm{H}), 3.71$ (s, 3H), $3.70(\mathrm{~s}, 3 \mathrm{H}) ;{ }^{13} \mathrm{C}$ NMR $\left(100 \mathrm{MHz}, \mathrm{CDCl}_{3}\right) \delta: 159.4$ $\left(\mathrm{d}, J_{\mathrm{C}-\mathrm{P}}=2.8 \mathrm{~Hz}\right), 136.3\left(\mathrm{~d}, J_{\mathrm{C}-\mathrm{P}}=4.2 \mathrm{~Hz}\right), 114.3\left(\mathrm{~d}, J_{\mathrm{C}-\mathrm{P}}=\right.$ $2.4 \mathrm{~Hz}), 112.0\left(\mathrm{~d}, J_{\mathrm{C}-\mathrm{P}}=8.7 \mathrm{~Hz}\right), 54.3,52.9\left(\mathrm{~d}, J_{\mathrm{C}-\mathrm{P}}=5.7\right.$ $\mathrm{Hz}) ;{ }^{31} \mathrm{P}$ NMR (162 MHz, $\left.\mathrm{CDCl}_{3}\right) \delta: 22.2$.

$O, O$-二甲基- $S e$-对叔丁基苯基磷酸酯(4d): 洗脱剂: $V$ (石油梄) $： V$ (乙酸乙酯 $)=1: 1$, 无色油状液体. $56 \mathrm{mg}$, 产率 $88 \% .{ }^{1} \mathrm{H}$ NMR $\left(400 \mathrm{MHz}, \mathrm{CDCl}_{3}\right) \delta: 7.47$ (dd, $J=8.5$ 
$\mathrm{Hz}, 2 \mathrm{H}), 7.26$ (d, $J=8.5 \mathrm{~Hz}, 2 \mathrm{H}), 3.75$ (s, 3H), 3.71 (s, $3 \mathrm{H}), 1.23$ (s, 9H); ${ }^{13} \mathrm{C}$ NMR (100 MHz, $\left.\mathrm{CDCl}_{3}\right) \delta: 151.2$ $\left(\mathrm{d}, J_{\mathrm{C}-\mathrm{P}}=2.9 \mathrm{~Hz}\right), 134.3\left(\mathrm{~d}, J_{\mathrm{C}-\mathrm{P}}=4.4 \mathrm{~Hz}\right), 125.8\left(\mathrm{~d}, J_{\mathrm{C}-\mathrm{P}}=\right.$ $2.3 \mathrm{~Hz}), 118.5\left(\mathrm{~d}, J_{\mathrm{C}-\mathrm{P}}=8.6 \mathrm{~Hz}\right), 52.9\left(\mathrm{~d}, J_{\mathrm{C}-\mathrm{P}}=5.6 \mathrm{~Hz}\right)$, $30.2 ;{ }^{31} \mathrm{P}$ NMR $\left(162 \mathrm{MHz}, \mathrm{CDCl}_{3}\right) \delta: 22.3$. HRMS (ESI) calcd for $\mathrm{C}_{12} \mathrm{H}_{19} \mathrm{O}_{3} \mathrm{PSe}[\mathrm{M}+\mathrm{H}]^{+}$323.0310, found 323.0323 .

$O, O$-二甲基-Se-对三氟甲氧基磷酸酯(4e): 洗脱剂: $V($ 石油醚 $): V($ 乙酸乙酯 $)=4: 1$, 无色油状液体. $64 \mathrm{mg}$, 产率 91\%. ${ }^{1} \mathrm{H}$ NMR (400 MHz, $\left.\mathrm{CDCl}_{3}\right) \delta: 7.60$ (dd, $J=8.8$ $\mathrm{Hz}, 2 \mathrm{H}), 7.10$ (d, $J=8.8 \mathrm{~Hz}, 2 \mathrm{H}), 3.75$ (s, 3H), 3.72 (s, $3 \mathrm{H}) ;{ }^{13} \mathrm{C}$ NMR $\left(100 \mathrm{MHz}, \mathrm{CDCl}_{3}\right) \delta: 151.32 \sim 145.90(\mathrm{~m})$, $136.1\left(\mathrm{~d}, J_{\mathrm{C}-\mathrm{P}}=4.6 \mathrm{~Hz}\right), 120.9\left(\mathrm{~d}, J_{\mathrm{C}-\mathrm{P}}=2.1 \mathrm{~Hz}\right), 120.4(\mathrm{~d}$, $\left.J_{\mathrm{C}-\mathrm{P}}=8.6 \mathrm{~Hz}\right), 119.3\left(\mathrm{q}, J_{\mathrm{C}-\mathrm{F}}=256.0 \mathrm{~Hz}\right), 53.1\left(\mathrm{~d}, J_{\mathrm{C}-\mathrm{P}}=5.9\right.$ $\mathrm{Hz}) ;{ }^{19} \mathrm{~F} \mathrm{NMR}\left(376 \mathrm{MHz}, \mathrm{CDCl}_{3}\right) \delta$ : $-57.9 ;{ }^{31} \mathrm{P} \mathrm{NMR}$ $\left(162 \mathrm{MHz}, \mathrm{CDCl}_{3}\right) \delta$ : 21.0. HRMS (ESI) calcd for $\mathrm{C}_{9} \mathrm{H}_{10} \mathrm{~F}_{3} \mathrm{O}_{4} \mathrm{PSe}[\mathrm{M}+\mathrm{H}]^{+}$350.9507, found 350.9530 .

$O, O$-二甲基- $S e$-2 - 䒜基苯基磷酸酯(4f $)^{[7 b]}$ : 洗脱剂: $V$ (石油醚) $: V$ (乙酸乙酯 $)=1: 1$, 黄色固体, $59 \mathrm{mg}$, 产 率 93\%, m.p. 55 56 ${ }^{\circ} \mathrm{C} ;{ }^{1} \mathrm{H}$ NMR $\left(400 \mathrm{MHz}, \mathrm{CDCl}_{3}\right) \delta$ : $8.38(\mathrm{~d}, J=8.4 \mathrm{~Hz}, 1 \mathrm{H}), 8.04 \sim 7.84(\mathrm{~m}, 1 \mathrm{H}), 7.86 \sim 7.80$ (m, 1H), $7.79 \sim 7.74(\mathrm{~m}, 1 \mathrm{H}), 7.53$ (ddd, $J=8.4,6.8,1.4$ $\mathrm{Hz}, 1 \mathrm{H}), 7.45$ (ddd, $J=8.1,6.9,1.2 \mathrm{~Hz}, 1 \mathrm{H}), 7.34$ (t, $J=$ $7.7 \mathrm{~Hz}, 1 \mathrm{H}), 3.66$ (s, 3H), 3.63 (s, 3H); ${ }^{13} \mathrm{C}$ NMR (100 $\left.\mathrm{MHz}, \mathrm{CDCl}_{3}\right) \delta: 135.5\left(\mathrm{~d}, J_{\mathrm{C}-\mathrm{P}}=4.9 \mathrm{~Hz}\right), 133.9\left(\mathrm{~d}, J_{\mathrm{C}-\mathrm{P}}=\right.$ $3.3 \mathrm{~Hz}), 133.2\left(\mathrm{~d}, J_{\mathrm{C}-\mathrm{P}}=2.2 \mathrm{~Hz}\right), 129.4\left(\mathrm{~d}, J_{\mathrm{C}-\mathrm{P}}=3.2 \mathrm{~Hz}\right)$, 127.7, 126.9, 126.2, 125.5, $124.9\left(\mathrm{~d}, J_{\mathrm{C}-\mathrm{P}}=3.2 \mathrm{~Hz}\right), 121.6$ $\left(\mathrm{d}, J_{\mathrm{C}-\mathrm{P}}=9.4 \mathrm{~Hz}\right), 53.1\left(\mathrm{~d}, J_{\mathrm{C}-\mathrm{P}}=6.0 \mathrm{~Hz}\right) ;{ }^{31} \mathrm{P}$ NMR $(162$ $\left.\mathrm{MHz}, \mathrm{CDCl}_{3}\right) \delta: 21.1$.

$O, O$-二甲基- $S e-2$-噻吩基磷酸酯(4g): 洗脱剂: $V$ (石 油醚) $: V$ (乙酸乙酯 $)=2: 1$, 无色油状液体. $38 \mathrm{mg}$, 产 率 70\%. ${ }^{1} \mathrm{H}$ NMR $\left(400 \mathrm{MHz}, \mathrm{CDCl}_{3}\right) \delta: 7.39$ (ddd, $J=5.4$, 2.6, $1.2 \mathrm{~Hz}, 1 \mathrm{H}), 7.20(\mathrm{td}, J=3.3,1.2 \mathrm{~Hz}, 1 \mathrm{H}), 6.95(\mathrm{dd}$, $J=5.3,3.6 \mathrm{~Hz}, 1 \mathrm{H}), 3.77$ (s, $3 \mathrm{H}), 3.74$ (s, $3 \mathrm{H}) ;{ }^{13} \mathrm{C} \mathrm{NMR}$ $\left(100 \mathrm{MHz}, \mathrm{CDCl}_{3}\right) \delta: 136.3\left(\mathrm{~d}, J_{\mathrm{C}-\mathrm{P}}=5.8 \mathrm{~Hz}\right), 131.1(\mathrm{~d}$, $\left.J_{\mathrm{C}-\mathrm{P}}=3.8 \mathrm{~Hz}\right), 127.3\left(\mathrm{~d}, J_{\mathrm{C}-\mathrm{P}}=3.3 \mathrm{~Hz}\right), 114.7\left(\mathrm{~d}, J_{\mathrm{C}-\mathrm{P}}=10.5\right.$ $\mathrm{Hz}), 53.2\left(\mathrm{~d}, J_{\mathrm{C}-\mathrm{P}}=5.5 \mathrm{~Hz}\right) ;{ }^{31} \mathrm{P} \mathrm{NMR}\left(162 \mathrm{MHz}, \mathrm{CDCl}_{3}\right)$ $\delta$ : 20.2. HRMS (ESI) calcd for $\mathrm{C}_{6} \mathrm{H}_{9} \mathrm{O}_{3} \mathrm{PSSe}[\mathrm{M}+\mathrm{H}]^{+}$ 272.9248, found 272.9263 .

$O, O$-二甲基- $S e$-1-(3,4-亚甲基二氧基)苯基磷酸酯 $(\mathbf{4 h})$ : 洗脱剂: $V$ (石油醚) $: V$ (乙酸乙酯 $)=2: 1$. 无色油 状液体. $48 \mathrm{mg}$, 产率 77\%. ${ }^{1} \mathrm{H}$ NMR $\left(400 \mathrm{MHz}, \mathrm{CDCl}_{3}\right) \delta$ : $7.21 \sim 7.02(\mathrm{~m}, 2 \mathrm{H}), 6.77(\mathrm{~d}, J=8.0 \mathrm{~Hz}, 1 \mathrm{H}), 5.99(\mathrm{~s}, 2 \mathrm{H})$, $3.82(\mathrm{~s}, 3 \mathrm{H}), 3.79$ (s, $3 \mathrm{H}) ;{ }^{13} \mathrm{C} \mathrm{NMR}\left(100 \mathrm{MHz}, \mathrm{CDCl}_{3}\right) \delta$ : $148.8\left(\mathrm{~d}, J_{\mathrm{C}-\mathrm{P}}=3.0 \mathrm{~Hz}\right), 148.4\left(\mathrm{~d}, J_{\mathrm{C}-\mathrm{P}}=2.5 \mathrm{~Hz}\right), 130.1(\mathrm{~d}$,
$\left.J_{\mathrm{C}-\mathrm{P}}=5.3 \mathrm{~Hz}\right), 113.9\left(\mathrm{~d}, J_{\mathrm{C}-\mathrm{P}}=9.0 \mathrm{~Hz}\right), 109.4\left(\mathrm{~d}, J_{\mathrm{C}-\mathrm{P}}=2.7\right.$ $\mathrm{Hz}), 101.9,54.0\left(\mathrm{~d}, J_{\mathrm{C}-\mathrm{P}}=5.7 \mathrm{~Hz}\right) ;{ }^{31} \mathrm{P}$ NMR $(162 \mathrm{MHz}$, $\left.\mathrm{CDCl}_{3}\right) \delta$ : 22.2 HRMS (ESI) calcd for $\mathrm{C}_{9} \mathrm{H}_{11} \mathrm{O}_{5} \mathrm{PSe}[\mathrm{M}+$ $\mathrm{H}^{+}$310.9582, found 310.9593 .

$O, O$-二苯基- $S e$-甲基磷酸酯 $(4 \mathbf{i})^{[18]}$ : 洗脱剂: $V\left(\right.$ 石油 $^{\prime}$ 醚) $: V$ (乙酸乙酯 $)=4: 1$, 黄色油状液体. $44 \mathrm{mg}$, 产率 $71 \%$. ${ }^{1} \mathrm{H}$ NMR $\left(400 \mathrm{MHz}, \mathrm{CDCl}_{3}\right) \delta: 7.39 \sim 7.18(\mathrm{~m}, 8 \mathrm{H})$, $7.19 \sim 7.08$ (m, 2H), 2.13 (d, $\left.J_{\mathrm{C}-\mathrm{P}}=14.8 \mathrm{~Hz}, 3 \mathrm{H}\right),{ }^{13} \mathrm{C} \mathrm{NMR}$ $\left(100 \mathrm{MHz}, \mathrm{CDCl}_{3}\right) \delta: 150.1\left(\mathrm{~d}, J_{\mathrm{C}-\mathrm{P}}=8.1 \mathrm{~Hz}\right), 129.9(\mathrm{~d}$, $\left.J_{\mathrm{C}-\mathrm{P}}=1.4 \mathrm{~Hz}\right), 125.7\left(\mathrm{~d}, J_{\mathrm{C}-\mathrm{P}}=1.7 \mathrm{~Hz}\right), 120.8\left(\mathrm{~d}, J_{\mathrm{C}-\mathrm{P}}=5.0\right.$ $\mathrm{Hz}), 5.7\left(\mathrm{~d}, J_{\mathrm{C}-\mathrm{P}}=5.5 \mathrm{~Hz}\right) ;{ }^{31} \mathrm{P} \mathrm{NMR}\left(162 \mathrm{MHz}, \mathrm{CDCl}_{3}\right) \delta$ : 13.4 .

$O, O$-二苯基- $S e$-异丁基磷酸酯 $(\mathbf{4} \mathbf{j})$ ：洗脱剂： $V$ (石油 醚) $: V$ (乙酸乙酯 $)=10: 1$, 无色油状液体. $46 \mathrm{mg}$, 产率 $60 \% .{ }^{1} \mathrm{H}$ NMR $\left(400 \mathrm{MHz}, \mathrm{CDCl}_{3}\right) \delta: 7.40 \sim 7.34(\mathrm{~m}, 3 \mathrm{H})$, $7.31(\mathrm{dt}, J=8.7,1.4 \mathrm{~Hz}, 3 \mathrm{H}), 7.28 \sim 7.20(\mathrm{~m}, 4 \mathrm{H}), 2.88$ (dd, $J=13.9,6.7 \mathrm{~Hz}, 2 \mathrm{H}), 1.83(\mathrm{dt}, J=13.3,6.7 \mathrm{~Hz}, 1 \mathrm{H})$, 0.93 (s, 3H), 0.91 (s, 3H); ${ }^{13} \mathrm{C}$ NMR $\left(100 \mathrm{MHz}, \mathrm{CDCl}_{3}\right) \delta$ : $150.1\left(\mathrm{~d}, J_{\mathrm{C}-\mathrm{P}}=8.2 \mathrm{~Hz}\right), 129.8\left(\mathrm{~d}, J_{\mathrm{C}-\mathrm{P}}=1.4 \mathrm{~Hz}\right), 125.6(\mathrm{~d}$, $\left.J_{\mathrm{C}-\mathrm{P}}=1.7 \mathrm{~Hz}\right), 120.9\left(\mathrm{~d}, J_{\mathrm{C}-\mathrm{P}}=5.0 \mathrm{~Hz}\right), 37.0\left(\mathrm{~d}, J_{\mathrm{C}-\mathrm{P}}=4.8\right.$ $\mathrm{Hz}), 29.6\left(\mathrm{~d}, J_{\mathrm{C}-\mathrm{P}}=18.6 \mathrm{~Hz}\right), 22.2 ;{ }^{31} \mathrm{P}$ NMR $(162 \mathrm{MHz}$, $\mathrm{CDCl}_{3}$ ) $\delta$ : 14.2. HRMS (ESI) calcd for $\mathrm{C}_{16} \mathrm{H}_{19} \mathrm{O}_{3} \mathrm{PSe}[\mathrm{M}+$ $\mathrm{H}]^{+} 371.0310$, found 371.0322 .

$O, O$-二苯基- $S e$-正戊基磷酸酯(4k): 洗脱剂: $V$ (石油 醚) $: V$ (乙酸乙酯 $)=12: 1$, 黄色油状液体. $49 \mathrm{mg}$, 产率 64\%. ${ }^{1} \mathrm{H}$ NMR (400 MHz, $\left.\mathrm{CDCl}_{3}\right) \delta: 7.41 \sim 7.34(\mathrm{~m}, 4 \mathrm{H})$, $7.34 \sim 7.28(\mathrm{~m}, 4 \mathrm{H}), 7.27 \sim 7.19(\mathrm{~m}, 2 \mathrm{H}), 2.97 \sim 2.90(\mathrm{~m}$, $2 \mathrm{H}), 1.67 \sim 1.62(\mathrm{~m}, 2 \mathrm{H}), 1.35 \sim 1.14(\mathrm{~m}, 4 \mathrm{H}), 0.84(\mathrm{t}, J=$ $8.0 \mathrm{~Hz}, 3 \mathrm{H}) ;{ }^{13} \mathrm{C}$ NMR $\left(100 \mathrm{MHz}, \mathrm{CDCl}_{3}\right) \delta: 150.2(\mathrm{~d}$, $\left.J_{\mathrm{C}-\mathrm{P}}=8.2 \mathrm{~Hz}\right), 129.8\left(\mathrm{~d}, J_{\mathrm{C}-\mathrm{P}}=1.4 \mathrm{~Hz}\right), 125.6\left(\mathrm{~d}, J_{\mathrm{C}-\mathrm{P}}=1.9\right.$ $\mathrm{Hz}), 120.9\left(\mathrm{~d}, J_{\mathrm{C}-\mathrm{P}}=4.9 \mathrm{~Hz}\right), 31.6,30.7\left(\mathrm{~d}, J_{\mathrm{C}-\mathrm{P}}=4.9 \mathrm{~Hz}\right)$, $28.0\left(\mathrm{~d}, J_{\mathrm{C}-\mathrm{P}}=4.8 \mathrm{~Hz}\right), 22.0,13.9 ;{ }^{31} \mathrm{P}$ NMR $(162 \mathrm{MHz}$, $\mathrm{CDCl}_{3}$ ) $\delta$ : 14.1. HRMS (ESI) calcd for $\mathrm{C}_{17} \mathrm{H}_{21} \mathrm{O}_{3} \mathrm{PSe}[\mathrm{M}+$ $\mathrm{H}]^{+} 385.0466$, found 385.0440 .

$O, O$-二苯基-Se-正庚基磷酸酯(4I): 洗脱剂: $V$ (石油 醚) $: V$ (乙酸乙酯 $)=10: 1$, 无色油状液体. $59 \mathrm{mg}$, 产率 $72 \% .{ }^{1} \mathrm{H}$ NMR (400 MHz, $\left.\mathrm{CDCl}_{3}\right) \delta: 7.31 \sim 7.27(\mathrm{~m}, 4 \mathrm{H})$, $7.25 \sim 7.22(\mathrm{~m}, 4 \mathrm{H}), 7.21 \sim 7.13(\mathrm{~m}, 2 \mathrm{H}), 2.86(\mathrm{dt}, J=$ 14.7, $7.4 \mathrm{~Hz}, 2 \mathrm{H}), 1.57 \sim 1.54(\mathrm{~m}, 2 \mathrm{H}), 1.44 \sim 0.98(\mathrm{~m}$, $8 \mathrm{H}), 0.79$ (t, $J=7.0 \mathrm{~Hz}, 3 \mathrm{H}) ;{ }^{13} \mathrm{C}$ NMR $\left(100 \mathrm{MHz}, \mathrm{CDCl}_{3}\right)$ $\delta: 150.2\left(\mathrm{~d}, J_{\mathrm{C}-\mathrm{P}}=8.2 \mathrm{~Hz}\right), 129.8\left(\mathrm{~d}, J_{\mathrm{C}-\mathrm{P}}=1.4 \mathrm{~Hz}\right), 125.6$ $\left(\mathrm{d}, J_{\mathrm{C}-\mathrm{P}}=1.7 \mathrm{~Hz}\right), 120.9\left(\mathrm{~d}, J_{\mathrm{C}-\mathrm{P}}=4.9 \mathrm{~Hz}\right), 31.6,31.0(\mathrm{~d}$, $\left.J_{\mathrm{C}-\mathrm{P}}=4.8 \mathrm{~Hz}\right), 29.4,28.6,28.0\left(\mathrm{~d}, J_{\mathrm{C}-\mathrm{P}}=4.8 \mathrm{~Hz}\right), 22.6$, $14.1 ;{ }^{31} \mathrm{P}$ NMR $\left(162 \mathrm{MHz}, \mathrm{CDCl}_{3}\right) \delta: 14.1$. HRMS (ESI) calcd for $\mathrm{C}_{19} \mathrm{H}_{25} \mathrm{O}_{3}$ PSe $[\mathrm{M}+\mathrm{H}]^{+}$413.0779, found 
413.0795.

$O, O$-二苯基- $S e$-环己基磷酸酯 $(4 \mathrm{~m})$ : 洗脱剂: $V$ (石 油醚) $: V$ (乙酸乙酯 $)=8: 1$, 无色油状液体. $58 \mathrm{mg}$, 产 率 $73 \% .{ }^{1} \mathrm{H}$ NMR $\left(400 \mathrm{MHz}, \mathrm{CDCl}_{3}\right) \delta: 7.38 \sim 7.32(\mathrm{~m}$, $4 \mathrm{H}), 7.33 \sim 7.25(\mathrm{~m}, 4 \mathrm{H}), 7.24 \sim 7.20(\mathrm{~m}, 2 \mathrm{H}), 3.79 \sim 3.54$ $(\mathrm{m}, 1 \mathrm{H}), 2.19 \sim 1.97(\mathrm{~m}, 2 \mathrm{H}), 1.67 \sim 1.47(\mathrm{~m}, 5 \mathrm{H}), 1.44 \sim$ $1.26(\mathrm{~m}, 3 \mathrm{H}) ;{ }^{13} \mathrm{C}$ NMR (100 MHz, $\left.\mathrm{CDCl}_{3}\right) \delta: 150.3(\mathrm{~d}$, $\left.J_{\mathrm{C}-\mathrm{P}}=8.5 \mathrm{~Hz}\right), 129.7\left(\mathrm{~d}, J_{\mathrm{C}-\mathrm{P}}=1.3 \mathrm{~Hz}\right), 125.5\left(\mathrm{~d}, J_{\mathrm{C}-\mathrm{P}}=1.9\right.$ $\mathrm{Hz}), 121.0\left(\mathrm{~d}, J_{\mathrm{C}-\mathrm{P}}=4.8 \mathrm{~Hz}\right), 46.5\left(\mathrm{~d}, J_{\mathrm{C}-\mathrm{P}}=4.3 \mathrm{~Hz}\right), 35.5$ $\left(\mathrm{d}, J_{\mathrm{C}-\mathrm{P}}=5.6 \mathrm{~Hz}\right), 26.5,25.3 ;{ }^{31} \mathrm{P} \mathrm{NMR}\left(162 \mathrm{MHz}, \mathrm{CDCl}_{3}\right)$ $\delta: 14.2$. HRMS (ESI) calcd for $\mathrm{C}_{18} \mathrm{H}_{21} \mathrm{O}_{3}$ PSe $[\mathrm{M}+\mathrm{H}]^{+}$ 397.0466, found 397.0480.

$O, O$-二苯基- $S e$-苄基磷酸酯 $(\mathbf{4 n})^{[9 \mathrm{a}]}$ : 洗脱剂: $V$ (石油 醚) $: V($ 乙酸乙酯 $)=10: 1$, 黄色固体. $49 \mathrm{mg}$, 产率 $61 \%$, m.p. 53 54 ${ }^{\circ} \mathrm{C} ;{ }^{1} \mathrm{H}$ NMR (400 MHz, $\left.\mathrm{CDCl}_{3}\right) \delta$ : $7.31 \sim 7.24(\mathrm{~m}, 4 \mathrm{H}), 7.20 \sim 7.09(\mathrm{~m}, 11 \mathrm{H}), 4.07(\mathrm{~d}, J=$ $12.6 \mathrm{~Hz}, 2 \mathrm{H}) ;{ }^{13} \mathrm{C} \mathrm{NMR}\left(100 \mathrm{MHz}, \mathrm{CDCl}_{3}\right) \delta: 149.0(\mathrm{~d}$, $\left.J_{\mathrm{C}-\mathrm{P}}=8.3 \mathrm{~Hz}\right), 136.3\left(\mathrm{~d}, J_{\mathrm{C}-\mathrm{P}}=5.9 \mathrm{~Hz}\right), 128.8\left(\mathrm{~d}, J_{\mathrm{C}-\mathrm{P}}=1.4\right.$ $\mathrm{Hz}), 128.0,127.7,126.6,124.6$ (d, $\left.J_{\mathrm{C}-\mathrm{P}}=1.7 \mathrm{~Hz}\right), 119.9$ (d, $\left.J_{\mathrm{C}-\mathrm{P}}=5.0 \mathrm{~Hz}\right), 29.7\left(\mathrm{~d}, J_{\mathrm{C}-\mathrm{P}}=4.8 \mathrm{~Hz}\right) ;{ }^{31} \mathrm{P}$ NMR $(162 \mathrm{MHz}$, $\left.\mathrm{CDCl}_{3}\right) \delta: 13.4$.

$\mathrm{O}, \mathrm{O}$-二苯基- $\mathrm{Se}$-苯乙基磷酸酯(4o): 洗脱剂: $V$ (石油 醚) $: V($ 乙酸乙酯 $)=10 ： 1$, 无色油状液体. $54 \mathrm{mg}$, 产率 $65 \% .{ }^{1} \mathrm{H}$ NMR (400 MHz, $\left.\mathrm{CDCl}_{3}\right) \delta: 7.40 \sim 7.36(\mathrm{~m}, 4 \mathrm{H})$, $7.34 \sim 7.28(\mathrm{~m}, 4 \mathrm{H}), 7.27 \sim 7.19(\mathrm{~m}, 5 \mathrm{H}), 7.11 \sim 7.05(\mathrm{~m}$, 2H), $3.30 \sim 3.07(\mathrm{~m}, 2 \mathrm{H}), 2.95 \sim 2.91(\mathrm{~m}, 2 \mathrm{H}) ;{ }^{13} \mathrm{C} \mathrm{NMR}$ $\left(100 \mathrm{MHz}, \mathrm{CDCl}_{3}\right) \delta: 150.1\left(\mathrm{~d}, J_{\mathrm{C}-\mathrm{P}}=8.2 \mathrm{~Hz}\right), 140.8$, 139.9, $129.7\left(\mathrm{~d}, J_{\mathrm{C}-\mathrm{P}}=1.3 \mathrm{~Hz}\right), 128.6\left(\mathrm{~d}, J_{\mathrm{C}-\mathrm{P}}=9.7 \mathrm{~Hz}\right)$, $128.5\left(\mathrm{~d}, J_{\mathrm{C}-\mathrm{P}}=3.7 \mathrm{~Hz}\right), 126.7,126.4,125.7\left(\mathrm{~d}, J_{\mathrm{C}-\mathrm{P}}=1.7\right.$ $\mathrm{Hz}), 121.0$ (d, $\left.J_{\mathrm{C}-\mathrm{P}}=4.9 \mathrm{~Hz}\right), 37.4\left(\mathrm{~d}, J_{\mathrm{C}-\mathrm{P}}=4.6 \mathrm{~Hz}\right), 28.5$ $\left(\mathrm{d}, J_{\mathrm{C}-\mathrm{P}}=4.6 \mathrm{~Hz}\right) ;{ }^{31} \mathrm{P} \mathrm{NMR}\left(162 \mathrm{MHz}, \mathrm{CDCl}_{3}\right) \delta: 13.8$. HRMS (ESI) calcd for $\mathrm{C}_{20} \mathrm{H}_{19} \mathrm{O}_{3} \mathrm{PSe}[\mathrm{M}+\mathrm{H}]^{+} 419.0310$, found 419.0329 .

$O, O$-二苯基- $S e-2$-(2-甲氧基)丙基磷酸酯(4p): 洗脱 剂: $V$ (石油醚) $: V$ (乙酸乙酯 $)=12: 1$, 黄色油状液体. $59 \mathrm{mg}$, 产率 77\%. ${ }^{1} \mathrm{H}$ NMR $\left(400 \mathrm{MHz}, \mathrm{CDCl}_{3}\right) \delta: 7.39 \sim$ $7.35(\mathrm{~m}, 4 \mathrm{H}), 7.33 \sim 7.26(\mathrm{~m}, 4 \mathrm{H}), 7.26 \sim 7.19(\mathrm{~m}, 2 \mathrm{H})$, $3.62(\mathrm{~s}, 3 \mathrm{H}), 1.81(\mathrm{~s}, 6 \mathrm{H}) ;{ }^{13} \mathrm{C} \mathrm{NMR}\left(100 \mathrm{MHz}, \mathrm{CDCl}_{3}\right) \delta$ : $173.7\left(\mathrm{~d}, J_{\mathrm{C}-\mathrm{P}}=4.3 \mathrm{~Hz}\right), 150.1\left(\mathrm{~d}, J_{\mathrm{C}-\mathrm{P}}=9.2 \mathrm{~Hz}\right), 129.8(\mathrm{~d}$, $\left.J_{\mathrm{C}-\mathrm{P}}=1.3 \mathrm{~Hz}\right), 125.6\left(\mathrm{~d}, J_{\mathrm{C}-\mathrm{P}}=1.7 \mathrm{~Hz}\right), 120.9\left(\mathrm{~d}, J_{\mathrm{C}-\mathrm{P}}=4.9\right.$ $\mathrm{Hz}), 53.0,51.3\left(\mathrm{~d}, J_{\mathrm{C}-\mathrm{P}}=5.3 \mathrm{~Hz}\right), 28.4\left(\mathrm{~d}, J_{\mathrm{C}-\mathrm{P}}=6.4 \mathrm{~Hz}\right)$; ${ }^{31} \mathrm{P}$ NMR (162 MHz, $\left.\mathrm{CDCl}_{3}\right) \delta$ : 9.5. HRMS (ESI) calcd for $\mathrm{C}_{16} \mathrm{H}_{19} \mathrm{O}_{4} \mathrm{PSe}[\mathrm{M}+\mathrm{H}]^{+}$387.0259, found 387.0269.

$O, O$-二苠基- $T e$-苯基磷酸酯(4q) ${ }^{[1 \mathrm{~d}]}$ : 洗脱剂: $V$ (石油 醚) $: V($ 乙酸乙酯 $)=5: 1$, 暗红色油状液体. $54 \mathrm{mg}$, 产
率 58\%. ${ }^{1} \mathrm{H}$ NMR $\left(400 \mathrm{MHz}, \mathrm{CDCl}_{3}\right) \delta: 7.68(\mathrm{~d}, J=8.0$ $\mathrm{Hz}, 2 \mathrm{H}), 7.34 \sim 7.22(\mathrm{~m}, 7 \mathrm{H}), 7.21 \sim 7.16(\mathrm{~m}, 4 \mathrm{H}), 7.10(\mathrm{t}$, $J=7.6 \mathrm{~Hz}, 2 \mathrm{H}), 5.01(\mathrm{~d}, J=3.0 \mathrm{~Hz}, 2 \mathrm{H}), 4.99$ (d, $J=3.4$ $\mathrm{Hz}, 2 \mathrm{H}) ;{ }^{13} \mathrm{C}$ NMR $\left(100 \mathrm{MHz}, \mathrm{CDCl}_{3}\right) \delta: 139.1$ (d, $J_{\mathrm{C}-\mathrm{P}}=$ $4.0 \mathrm{~Hz}), 134.2\left(\mathrm{~d}, J_{\mathrm{C}-\mathrm{P}}=7.9 \mathrm{~Hz}\right), 128.6\left(\mathrm{~d}, J_{\mathrm{C}-\mathrm{P}}=2.1 \mathrm{~Hz}\right)$, $128.0\left(\mathrm{~d}, J_{\mathrm{C}-\mathrm{P}}=2.5 \mathrm{~Hz}\right), 127.5\left(\mathrm{~d}, J_{\mathrm{C}-\mathrm{P}}=2.5 \mathrm{~Hz}\right), 127.0$, $107.5\left(\mathrm{~d}, J_{\mathrm{C}-\mathrm{P}}=8.1 \mathrm{~Hz}\right), 67.7\left(\mathrm{~d}, J_{\mathrm{C}-\mathrm{P}}=5.9 \mathrm{~Hz}\right) ;{ }^{31} \mathrm{P}$ NMR $\left(162 \mathrm{MHz} \mathrm{CDCl}_{3}\right) \delta:-1.8$.

$O, O$-二正丁基- $T e$-苯基磷酸酯(4r) ${ }^{[1 \mathrm{~d}]}$ : 洗脱剂: $V($ 石 油醚 $): V($ 乙酸乙酯 $)=1: 1$, 黄色固体. $42 \mathrm{mg}$, 产率 53\%, m.p. $110 \sim 112{ }^{\circ} \mathrm{C} ;{ }^{1} \mathrm{H}$ NMR (400 MHz, $\left.\mathrm{CDCl}_{3}\right) \delta$ : $7.76(\mathrm{~d}, J=8.1,1.5 \mathrm{~Hz}, 2 \mathrm{H}), 7.29(\mathrm{td}, J=7.4,1.5 \mathrm{~Hz}, 1 \mathrm{H})$, $7.23 \sim 7.03(\mathrm{~m}, 2 \mathrm{H}), 4.17 \sim 3.80(\mathrm{~m}, 4 \mathrm{H}), 1.73 \sim 1.42(\mathrm{~m}$, $4 \mathrm{H}), 1.33 \sim 1.07(\mathrm{~m}, 4 \mathrm{H}), 0.82(\mathrm{t}, J=7.4 \mathrm{~Hz}, 6 \mathrm{H}) ;{ }^{13} \mathrm{C}$ NMR (100 MHz, $\left.\mathrm{CDCl}_{3}\right) \delta: 139.9\left(\mathrm{~d}, J_{\mathrm{C}-\mathrm{P}}=4.0 \mathrm{~Hz}\right), 129.6$ $\left(\mathrm{d}, J_{\mathrm{C}-\mathrm{P}}=1.9 \mathrm{~Hz}\right), 128.9\left(\mathrm{~d}, J_{\mathrm{C}-\mathrm{P}}=2.2 \mathrm{~Hz}\right), 108.9\left(\mathrm{~d}, J_{\mathrm{C}-\mathrm{P}}=\right.$ $8.0 \mathrm{~Hz}), 67.2\left(\mathrm{~d}, J_{\mathrm{C}-\mathrm{P}}=6.1 \mathrm{~Hz}\right), 31.9\left(\mathrm{~d}, J_{\mathrm{C}-\mathrm{P}}=7.3 \mathrm{~Hz}\right)$, 18.8, 13.6; ${ }^{31} \mathrm{P}$ NMR $\left(162 \mathrm{MHz}, \mathrm{CDCl}_{3}\right) \delta$ : -0.9 .

$O, O$-二甲基- $T e$-苯基磷酸酯 $(\mathbf{4 s})^{[1 \mathrm{~d}]}$ : 洗脱剂: $V($ 石油 醚) $: V($ 乙酸乙酯 $)=3: 1$, 暗红色油状液体. $30 \mathrm{mg}$, 产 率 48\%. ${ }^{1} \mathrm{H} \mathrm{NMR}\left(400 \mathrm{MHz}, \mathrm{CDCl}_{3}\right) \delta: 7.76(\mathrm{dt}, J=8.1$, $1.5 \mathrm{~Hz}, 2 \mathrm{H}), 7.33 \sim 7.23(\mathrm{~m}, 1 \mathrm{H}), 7.18(\mathrm{t}, J=7.6 \mathrm{~Hz}, 2 \mathrm{H})$, $3.67(\mathrm{~s}, 3 \mathrm{H}), 3.63(\mathrm{~s}, 3 \mathrm{H}) ;{ }^{13} \mathrm{C} \mathrm{NMR}\left(100 \mathrm{MHz}, \mathrm{CDCl}_{3}\right) \delta$ : $139.0\left(\mathrm{~d}, J_{\mathrm{C}-\mathrm{P}}=3.9 \mathrm{~Hz}\right), 128.9\left(\mathrm{~d}, J_{\mathrm{C}-\mathrm{P}}=2.0 \mathrm{~Hz}\right), 128.0(\mathrm{~d}$, $\left.J_{\mathrm{C}-\mathrm{P}}=2.3 \mathrm{~Hz}\right), 107.3\left(\mathrm{~d}, J_{\mathrm{C}-\mathrm{P}}=8.1 \mathrm{~Hz}\right), 52.7\left(\mathrm{~d}, J_{\mathrm{C}-\mathrm{P}}=5.4\right.$ $\mathrm{Hz}) ;{ }^{31} \mathrm{P}$ NMR $\left(162 \mathrm{MHz}, \mathrm{CDCl}_{3}\right) \delta: 3.6$.

$O, O$-二甲基- $T e-2$-䒺基磷酸酯 $(\mathbf{4 t})$ : 洗脱剂: $V$ (石油 醚) $: V($ 乙酸乙酯 $)=1: 1$, 暗红色油状液体. $31 \mathrm{mg}$, 产 率 43\%. ${ }^{1} \mathrm{H}$ NMR $\left(400 \mathrm{MHz}, \mathrm{CDCl}_{3}\right) \delta: 8.30 \sim 8.11(\mathrm{~m}$, 2H), $7.84(\mathrm{~d}, J=8.2 \mathrm{~Hz}, 1 \mathrm{H}), 7.73(\mathrm{~d}, J=7.9 \mathrm{~Hz}, 1 \mathrm{H})$, $7.59 \sim 7.48(\mathrm{~m}, 1 \mathrm{H}), 7.48 \sim 7.41(\mathrm{~m}, 1 \mathrm{H}), 7.27(\mathrm{t}, J=7.6$ $\mathrm{Hz}, 1 \mathrm{H}), 3.60$ (s, 3H), 3.57 (s, 3H); ${ }^{13} \mathrm{C}$ NMR (100 MHz, $\left.\mathrm{CDCl}_{3}\right) \delta: 142.0\left(\mathrm{~d}, J_{\mathrm{C}-\mathrm{P}}=4.2 \mathrm{~Hz}\right), 136.5\left(\mathrm{~d}, J_{\mathrm{C}-\mathrm{P}}=2.5 \mathrm{~Hz}\right)$, $133.8\left(\mathrm{~d}, J_{\mathrm{C}-\mathrm{P}}=1.9 \mathrm{~Hz}\right), 132.5,130.8\left(\mathrm{~d}, J_{\mathrm{C}-\mathrm{P}}=2.8 \mathrm{~Hz}\right)$, 129.0, 127.3, 126.6, 126.5, 112.0 (d, $\left.J_{\mathrm{C}-\mathrm{P}}=8.6 \mathrm{~Hz}\right), 53.7$ $\left(\mathrm{d}, J_{\mathrm{C}-\mathrm{P}}=5.6 \mathrm{~Hz}\right) ;{ }^{31} \mathrm{P} \mathrm{NMR}\left(162 \mathrm{MHz}, \mathrm{CDCl}_{3}\right) \delta: 2.5$. HRMS (ESI) calcd for $\mathrm{C}_{12} \mathrm{H}_{13} \mathrm{O}_{3} \mathrm{PTe}[\mathrm{M}+\mathrm{H}]^{+}$366.9737, found 366.9743 .

2-苯硒基吲哚 $(\mathbf{5})^{[14]}$ : 洗脱剂: $V$ (石油醚)： $V$ (乙酸乙 酯 $)=10 ： 1$, 黄色固体. $19 \mathrm{mg}$, 产率 35\%, m.p. 135 $136{ }^{\circ} \mathrm{C} ;{ }^{1} \mathrm{H}$ NMR (400 MHz, $\left.\mathrm{CDCl}_{3}\right) \delta$ : 8.36 (brs, $\left.1 \mathrm{H}\right)$, 7.56 (d, $J=7.9 \mathrm{~Hz}, 1 \mathrm{H}), 7.40$ (d, $J=2.5 \mathrm{~Hz}, 1 \mathrm{H}), 7.36$ (d, $J=8.1 \mathrm{~Hz}, 1 \mathrm{H}), 7.23 \sim 7.13(\mathrm{~m}, 3 \mathrm{H}), 7.13 \sim 6.97(\mathrm{~m}, 4 \mathrm{H})$; ${ }^{13} \mathrm{C}$ NMR $\left(100 \mathrm{MHz}, \mathrm{CDCl}_{3}\right) \delta: 135.3,132.8,130.2,128.9$, 127.9, 127.6, 124.5, 121.9, 119.8, 119.3, 110.3, 97.1. 
苯基-2,4,6-三甲氧苯硒醚 $(6)^{[14]}$ : 洗脱剂： $V($ 石油 醚) $: V($ 乙酸乙酯 $)=25: 1$, 白色固体. $28 \mathrm{mg}$, 产率 $43 \%$. m.p. $103 \sim 105{ }^{\circ} \mathrm{C} ;{ }^{1} \mathrm{H}$ NMR (400 MHz, $\left.\mathrm{CDCl}_{3}\right) \delta$ : $7.41 \sim 6.70(\mathrm{~m}, 5 \mathrm{H}), 6.14(\mathrm{~s}, 2 \mathrm{H}), 3.79(\mathrm{~s}, 3 \mathrm{H}), 3.72(\mathrm{~s}$, $6 \mathrm{H}) ;{ }^{13} \mathrm{C}$ NMR $\left(100 \mathrm{MHz}, \mathrm{CDCl}_{3}\right) \delta: 161.9,160.9,132.5$, 127.7, 127.6, 124.2, 96.0, 90.1, 55.3, 54.4.

\section{辅助材料(Supporting Information) 化合物 3-6 的核磁} 图谱. 这些材料可以免费从本刊网站(http://sioc-journal. $\mathrm{cn} /$ )上下载.

\section{References}

[1] (a) Carrasco, N.; Caton-Williams, J.; Brandt, G.; Wang, S.; Huang, Z. Angew. Chem., Int. Ed. 2006, 45, 94.

(b) Mitra, S.; Mukherjee, S.; Sen, S. K.; Hajra, A. Bioorg. Med. Chem. Lett. 2014, 24, 2198.

(c) Dinsdale, D.; Verschoyle, R. D. Biochem. Pharmacol. 2001, 61, 493.

(d) Mailahn, D. H.; Iarocz, L. E. B.; Nobre, P. C.; Perin, G.; Sinott, A.; Pesarico, A. P.; Birmann, P. T.; Savegnago, L.; Silva, M. S. Eur. J. Med. Chem. 2021, 213, 113052.

[2] (a) Han, L.-B.; Choi, N.; Tanaka, M. J. Am. Chem. Soc. 1996, 118, 7000 .

(b) Lopin, C.; Gouhier, G.; Gautier, A.; Piettre, S. R. J. Org. Chem. 2003, 68, 9916.

(c) Lopin, C.; Gautier, A.; Gouhier, G.; Piettre, S. R. Tetrahedron Lett. 2000, 41, 10195.

[3] Petragnani, N.; Toscano, V. G.; De Moura Campos, M. Chem. Ber. 1968, 101,3070 .

[4] (a) Sun, K.; Wang, X.; Li, C.; Wang, H.; Li, L. Org. Chem. Front. 2020, 7, 3100 .

(b) Xu, Y.; Li, C.; Meng, J.; Huang, Y.; Fu, J.; Liu, B.; Liu, Y.; Chen, N. Chin. J. Org. Chem. 2021, 41, 1012 (in Chinese).

(许颖, 李晨, 孟建萍, 黄玉玲, 付纪源, 刘冰, 刘颖杰, 陈宁, 有机化学, 2021, 41, 1012.)

[5] (a) Gao, Y.-X.; Tang, G.; Cao, Y.; Zhao, Y.-F. Synthesis 2009, 1081. (b) Sun, K.; Wang, S.; Feng, R.; Zhang, Y.; Wang, X.; Zhang, Z.; Zhang, B. Org. Lett. 2019, 21, 2052.

[6] (a) Zhang, X.; Shi, Z.; Shao, C.; Zhao, J.; Wang, D.; Zhang, G.; Li, L. Eur. J. Org. Chem. 2017, 1884.

(b) Li, Y.; Chen, S.; Su, L.; Li, J.; Xu, X. Chin. J. Org. Chem. 2013, 33, 1999 (in Chinese).

(李媛媛, 陈四海, 苏柳, 李建华, 许新华, 有机化学, 2013, 33, 1999.) (c) Wang, J.; Wang, X.; Li, H.; Yan, J. J. Organomet. Chem. 2018 , 859,75

(d) Chen, S.; Chen, J.; Xu, X.; He, Y.; Yi, R.; Qiu, R. J. Organomet. Chem. 2016, 818, 123.

[7] (a) Choudhary, R.; Singh, P.; Bai, R.; Sharma, M. C.; Badsara, S. S. Org. Biomol. Chem. 2019, 17, 9757.

(b) Bhunia, S. K.; Das, P.; Jana, R. Org. Biomol. Chem. 2018, 16, 9243 .

(c) Wang, X.; Wang, Q.; Xue, Y.; Sun, K.; Wu, L.; Zhang, B. Chem. Commun. 2020, 56, 4436.

(d) Wang, X.; Guo, S.; Zhang, Y.; Zhang, Z.; Zhang, G.; Ye, Y.; Sun, K. Adv. Synth. Catal. 2021, 363, 3290.

[8] (a) Chen, D.-J.; Chen, Z.-C. J. Chem. Res. 2000, 370.

(b) Xu, Q.; Liang, C.-G.; Huang, X. Synth. Commun. 2003, 33, 2777.

[9] (a) Guo, S.; Li, S.; Zhang, Z.; Yan, W.; Cai, H. Tetrahedron Lett. 2020, 61, 151566 .

(b) Amri, N.; Wirth, T. Synlett 2020, 31, 1894.

[10] Clive, D. L. J.; Menchen, S. M. J. Org. Chem. 1980, 45, 2347.

[11] (a) Wang, P.-F.; Yi, W.; Ling, Y.; Ming, L.; Liu, G.-Q.; Zhao, Y. Chin. Chem. Lett. 2021, 32, 2587.

(b) Liu, G.-Q.; Yi, W.; Wang, P.-F.; Liu, J.; Ma, M.; Hao, D.-Y.; Ming, L.; Ling, Y. Green Chem. 2021, 23, 1840.

(c) Liang, Z.-P.; Yi, W.; Wang, P.-F.; Liu, G.-Q.; Ling, Y. J. Org. Chem. 2021, 86, 5292.

[12] (a) Barnes, I.; Hjorth, J.; Mihalopoulos, N. Chem. Rev. 2006, 106, 940 .

(b) Wu, X.-F.; Natte, K. Adv. Synth. Catal. 2016, 358, 336.

[13] (a) Shi, M.; Wang, B.-Y.; Li, J. Eur. J. Org. Chem. 2005, 759.

(b) Perin, G.; Santoni, P.; Barcellos, A. M.; Nobre, P. C.; Jacob, R. G.; Lenardão, E. J.; Santi, C. Eur. J. Org. Chem. 2018, 1224.

(c) Tiecco, M.; Testaferri, L.; Tingoli, M.; Chianelli, D.; Bartoli, D. Tetrahedron Lett. 1989, 30, 1417.

(d) Tiecco, M.; Testaferri, L.; Temperini, A.; Bagnoli, L.; Marini, F.; Santi, C. Synlett 2001, 1767.

[14] Zhang, Q.-B.; Ban, Y.-L.; Yuan, P.-F.; Peng, S.-J.; Fang, J.-G.; Wu, L.-Z.; Liu, Q. Green Chem. 2017, 19, 5559.

[15] (a) Gancarz, R. A.; Kice, J. L. Tetrahedron Lett. 1981, 22, 1661. (b) Shimizu, M.; Takeda, R.; Kuwajima, I. Tetrahedron Lett. 1979, 419.

[16] (a) Reich, H. J.; Willis, W. W., Jr.; Wollowitz, S. Tetrahedron Lett. 1982, 23, 3319.

(b) Kice, J. L.; McAfee, F.; Slebocka-Tilk, H. Tetrahedron Lett. 1982, 23, 3323.

(c) Kang, S. I.; Kice, J. L. J. Org. Chem. 1985, 50, 2968.

[17] Singh, D.; Deobald, A. M.; Camargo, L. R. S.; Tabarelli, G.; Rodrigues, O. E. D.; Braga, A. L. Org. Lett. 2010, 12, 3288.

[18] Loginova, E. I.; Nuretdinov, I. A.; Petrov, Y. A. Teor. Eksp. Khim. 1974, 10, 75 . 